is staged in the old, tennis-court style space of the Hôtel de Bourgogne, a theatre that figures prominently in Gobert's overall excavation of Cartesian theatrical influence (including the substance of the Bourguignons' antagonistic relationship to Molière, delightfully detailed in the previous section of the book). In the move from the play's premiere in this venue to its inaugural presentation at the Comédie-Française newly designed by François d'Orbay, we follow the shift typical of theatre in France at this time. From buildings in which audience and actors shared the same space (i.e. interacted), late seventeenth-century theatre gradually came to divide the spectator from the play, very much along the lines of strict Cartesianist mind-body separation. Again, Gobert does not claim that Descartes engineered any of this; rather, the spirit of the age, with Descartes's philosophical and mathematical influence, was moving inexorably toward such binary formations. While it is true that the reflective subject springs from the philosophy of Descartes, it is also true that the overdevelopment of this subject, into a mind thoroughly disconnected from its body, is a fundamentally anti-Cartesian proposal.

As it turns out, what Gobert is really charting in his book is a substantive shift in theatrical representation, and at the root of this shift-which was to influence all subsequent theatre in the so-called "Western" world-he locates Descartes. It is a bold and sweeping claim, but his research is sound and his reading of the philosopher's various texts is solid. If there is a fault to Gobert's project, it is to be found in his frequent and emphatic proclamations pointing toward the strength of his claims about Descartes's influence. Such reminders are unnecessary in such a comprehensive and well-researched study; they only serve to diminish the rightful impact of this otherwise impressive work. Truly, one gets the sense in reading Gobert's book that he has completely exhausted the archive, and while a full one-third of the book is taken up by endnotes, the reader feels not exhausted by the author's thoroughness, but impassioned by it.

Book Editor's Note: R. Darren Gobert's The Mind-Body Stage is the 2013 recipient of the Canadian Association of Theatre Research's Ann Saddlemyer Award for best book in English or French.

\title{
SYLVAIN SCHRYBURT
}

\section{De l'acteur vedette au théâtre de festival. Histoire des pratiques scéniques montréalaises (1940-1980).}

Montréal : Les Presses de l'Université de Montréal, 2011. 395 pp.

\section{LOUISE LADOUCEUR}

Cette étude suit l'évolution du théâtre d'expression française à Montréal sur quatre décennies pour y mettre en relief les différents régimes qui ont marqué la pratique théâtrale au cours de ces années. Un des mérites de l'ouvrage est de dégager non seulement ce qui appartient en propre à chaque régime, mais les facteurs contribuant au changement de régime et la façon dont chacun s'articule au précédent pour ensuite modifier l'horizon d'attente du milieu théâ- 
tral et du public montréalais. Ce n'est plus une histoire "par morceaux» (II) mais un récit qui met les morceaux en place, jetant ainsi un nouvel éclairage sur leur articulation.

Louvrage est divisé en cinq chapitres. Le premier situe l'étude dans le champ historiographique du théâtre québécois, décrit la structure de l'ouvrage et présente les différents régimes théâtraux sur lesquels porte l'analyse. Les chapitres suivants sont consacrés à l'étude des trois régimes qui constituent des états successifs du champ théâtral montréalais marqués par des principes structurants distincts. Le premier régime, qui va de 1937 à 1952, est fondé sur l'acteur vedette : " hérité des pratiques du XIX ${ }^{\mathrm{e}}$ siècle et caractérisé par une approche commerciale du théâtre, centrée sur le vedettariat de l'acteur, l'absence de metteur en scène et le renouvellement hebdomadaire de l'affiche » (I6).Vers la fin de cette période, émerge un metteur en scène qui deviendra la figure structurante d'un nouveau régime qui couvre les années de I95I à 1960 et qui tend vers un «modèle de pureté »(9I). Ce régime se caractérise par « une rigueur accrue dans la conception des spectacles (par rapport à l'ancien régime), une centralisation des pouvoirs entre les mains du metteur en scène et un rythme de production espacé qui permet d'augmenter le temps alloué aux répétitions (I6). Avec la mise en place d'organisme subventionnaires, tel que le Conseil des Arts fondé en 1957, on passe d'un champ " embryonnaire " (I27) à une structuration construite sur une "dynamique de distinction" (I27) dans laquelle les compagnies bien établies vont se professionnaliser et affirmer leur spécialisation. Sous ce nouveau régime, qui va de 1958 à 1969 , les théâtres institutionnels, comprenant le Théâtre du Nouveau Monde et le Théâtre du Rideau-Vert, développent un jeu épuré privilégiant l'élégance du geste et vouent un culte au texte de haute tenue littéraire. Les véritables innovations viennent des troupes avant-garde de la marge, telles que le Théâtre des Saltimbanques et Les Apprentis-Sorciers. Ce théâtre amateur explore les ressources spatiales, visuelles et sonores de la scène dans des spectacles qui invitent à l'expérimentation. C'est l'époque où les théâtres de poche se multiplient, menant des recherches incessantes qui contribuent "à élargir l'espace des possibles du théâtre montréalais » (I68). Bousculant l'horizon d'attente du public montréalais, ces théâtres de la marge obligent les producteurs établis à dépasser les modèles qu'ils ont mis en place. Lentrée en scène du théâtre québécois coïncide avec un troisième régime, qualifié de « collectif » puisqu'il se définit par « un partage des pouvoirs entre les mains d'un groupe collectivement responsable de la production du sens du spectacle » (I6). Couvrant la période I968-I980, ce régime privilégie la création collective, qui se pratique surtout au sein des troupes non institutionnelles pendant que le metteur en scène devenu pigiste, et non plus associé à une seule troupe, affirme son art avec éclat. Lauteur analyse plus particulièrement les contributions de trois metteurs en scène : Paul Buissonneau, André Brassard et Jean-Pierre Ronfard. Il se penche aussi sur le fonctionnement de la troupe phare de la création collective, le Grand Cirque ordinaire, et sur le mouvement du Jeune Théâtre, dont quelques troupes d'extrême gauche engagées dans une recherche où « le politique prime sur le culturel " (309). Il analyse enfin la pratique d'autogestion propre au théâtre d'expérimentation et l'esthétique qui se dégage des derniers spectacles du Théâtre expérimental de Montréal. Lauteur conclut, finalement, à un nouveau régime structuré par les festivals, qui s'installe au début des années i980. Possédant son système de financement, le festival se réclame d'une esthétique qui lui est propre et opère une ouverture du théâtre québécois à la scène internationale.

Cette excellente étude s'appuie sur une riche documentation comprenant des archives 
sonores et audiovisuelles, des écrits et entretiens des praticiens, des archives journalistiques et des textes critiques. Elle propose une analyse nuancée, appuyée par d'abondantes ressources documentaires qui mettent en relief des aspects moins connus de l'histoire du théâtre qui se pratique à Montréal entre 1940 et 1980 . Finaliste pour un Prix du Canada en sciences humaines de la Fédération canadienne des sciences humaines en 2013, l'auteur propose avec cet ouvrage une lecture fascinante d'une époque effervescente de l'histoire théâtrale et culturelle montréalaise.

\section{MICHELLE MACARTHUR, LYDIA WILKINSON, and KEREN ZAIONTZ, eds. \\ Performing Adaptations: Essays \& Conversations on the Theory and Practice of Adaptation.}

Newcastle upon Tyne: Cambridge Scholars Publishing, 2009. 273 pp.

YANA MEERZON

The title of the edited collection, Performing Adaptations: Essays do Conversations on the Theory and Practice of Adaptation, promises an unusual format for an English-language scholarly publication. It suggests a free assembly of scholarly essays, conversations, interviews, artistic statements, and other materials related to the subject. Although such a format allows for a more creative approach, it also presents a challenge, specifically when a book is edited by emerging scholars. The co-editors, however, managed the task elegantly by selecting high quality articles and securing contributions from well-known and established scholars, such as Linda Hutcheon, Stephen Johnson, and Marlis Schweitzer, among others, as well as artistic statements from prolific American and Canadian theatre artists, experts in methodologies of adaptation for theatre, such as Tony Perucci, Marjorie Chan, George Elliott Clarke, and John Greyson, to name a few.

As the co-editors explain, the book stems from the 2006 Festival of Original Theatre: Adaptation in Performance, organized by the Graduate Centre for Study of Drama at the University of Toronto. Normally a collection of articles based on oral contributions presented at an academic gathering resembles conference proceedings. This book avoids that pitfall. It presents a well-balanced and thoughtful collection of contributions that complement one another. Specifically I would like to mention Stephen Johnson's article on Edwin S. Porter's cinematic rendering of Uncle Tom's Cabin. This essay provides insight on Edwin S. Porter's cinematic style and his personal traditions of adaptation. It also analyzes the episodic and improvisatory nature of this film's narrative that seems to be "at odds with mainstream theatre practice" of the time (87). This contribution is followed by two appendices, which also focus on theatrical adaptations of the novel. These chapters, however, appear to have been somewhat insubstantially contextualized by the co-editors. Publishing archival documents is a noble endeavour since public exposure to previously unknown material may lead to new and exciting theoretical inquiry. However, the book may fall short in this aspect unless the reader has studied multiple performance and cinematic renderings of Uncle Tom's Cabin.

The rest of the book presents a more successfully developed compilation. For instance, 